\title{
Finerenone: A Potential Treatment for Patients with Chronic Kidney Disease and Type 2 Diabetes Mellitus
}

Luis D’Marco, ${ }^{1,2}$ María Jesús Puchades, ${ }^{1,3}$ Lorena Gandía, ${ }^{1}$ Claudia Forquet, ${ }^{1}$ Elena Giménez-Civera, ${ }^{1}$ Nayara Panizo, ${ }^{1}$ Javier Reque, ${ }^{4}$ Isabel Juan-García, ${ }^{1}$ Valmore Bermúdez ${ }^{5}$ and José Luis Gorriz ${ }^{1,3}$

1. Nephrology Department, Hospital Clínico Universitario, Instituto de Investigación Sanitaria (INCLIVA), Valencia, Spain; 2. Centro de Estudios Universitarios Cardenal Herrera University, Valencia, Spain; 3. Valencia University, Valencia, Spain; 4. Nephrology Department, Hospital de Castellon, Castellon, Spain; 5. Facultad de Ciencias de la Salud, Universidad Simón Bolívar, Barranquilla, Colombia

\section{Keywords}

Chronic kidney disease, cardiovascular diseases,

diabetic nephropathies, finerenone

Disclosures: José Luis Gorriz has received speakers' fees and funding for attending advisory boards from Bayer. Luis D'Marco, María Jesús Puchades, Lorena Gandía, Claudia Forquet, Elena Giménez-Civera, Nayara Panizo, Javier Reque, Isabel Juan-García and Valmore Bermúdez have no financial or nonfinancial relationships or activities to declare in relation to this article.

Acknowledgements: Conceptualization: Luis D'Marco. Writing original draft: Luis D'Marco, Nayara Panizo, Javier Reque, Lorena Gandía, Claudia Forquet and Valmore Bermúdez. Figures: Luis D’Marco, María Jesús Puchades, Elena Giménez-Civera and Isabel Juan-García. Writing review and editing: Luis D’Marco, Valmore Bermúdez, José Luis Gorriz and María Jesús Puchades.

Review process: Double-blind peer review.

Compliance with ethics: This study involves a review of the literature and did not involve any studies with human or animal subjects performed by any of the authors.

Data availability: Data sharing is not applicable to this article as no

datasets were generated or analysed during the writing of this article.

Authorship: The named authors meet the International committee

of Medical Journal Editors (ICMJE) criteria for authorship of this

manuscript, take responsibility for the integrity of the work as a whole,

and have given final approval for the version to be published.

Access: This article is freely accessible at

touchENDOCRINOLOGY.com (c) Touch Medical Media 2021

Received: 29 June 2021

Accepted: 28 September 2021

Published online: 10 November 2021

Citation: touchREVIEWS in Endocrinology. 2021;17(2):84-7

Corresponding author: José Luis Gorriz, Nephrology Department, Hospital Clínico Universitario de Valencia, Av Blasco Ibanez 17, 46010 Valencia, Spain.

E: jlgorriz@gmail.com

Support: No funding was received in the publication of this article.
Chronic kidney disease (CKD) represents one of the greatest future public health challenges due, among other consequences, to its elevated burden on economic and human resources within healthcare systems. ${ }^{1}$ Moreover, the rapid growth of type 2 diabetes mellitus (T2DM) results in a high incidence of diabetic kidney disease (DKD), which, together with hypertension, is the main cause of CKD progression to end-stage kidney disease (ESKD) and the need for renal replacement therapies. ${ }^{2}$ The introduction of drugs such as sodium-glucose cotransporter 2 (SGLT-2) inhibitors and glucagon-like peptide 1 (GLP-1) receptor agonists has improved the ability to slow the progression of DKD, with additional benefits in cardiovascular disease (CVD);,3,4 however, the residual renal risk remains high and renal progression has not been completely arrested.

Beyond the effects of these novel antidiabetic drugs, a growing body of evidence suggests that the overactivation of the mineralocorticoid receptor also contributes to CKD progression. ${ }^{5}$ Thus, recent evidence supports the use of mineralocorticoid receptor antagonist (MRA) drugs with some therapeutic benefits. ${ }^{6}$ In this regard, findings from the FIDELIODKD (Finerenone in reducing kidney failure and disease progression in DKD) and more recently FIGARO-DKD (Finerenone in reducing cardiovascular mortality and morbidity in DKD) trials have demonstrated that the selective nonsteroidal MRA, finerenone, improves the risk of CKD progression and cardiovascular events in patients with CKD and T2DM and optimized renin-angiotensin-aldosterone system (RAAS) blockade. ${ }^{7,8}$ Moreover, finerenone also reduced albuminuria with minimal effects on blood pressure and glycaemia. Here, we review the rationale for the development and use of MRA drugs to slow CKD progression in patients with T2DM and DKD, as well as other pleiotropic effects, and highlight the warnings associated with these agents (Figure 1). 
Figure 1: Beneficial effects and warnings of mineralocorticoid receptor antagonist drugs in patients with chronic kidney disease and type 2 diabetes mellitus

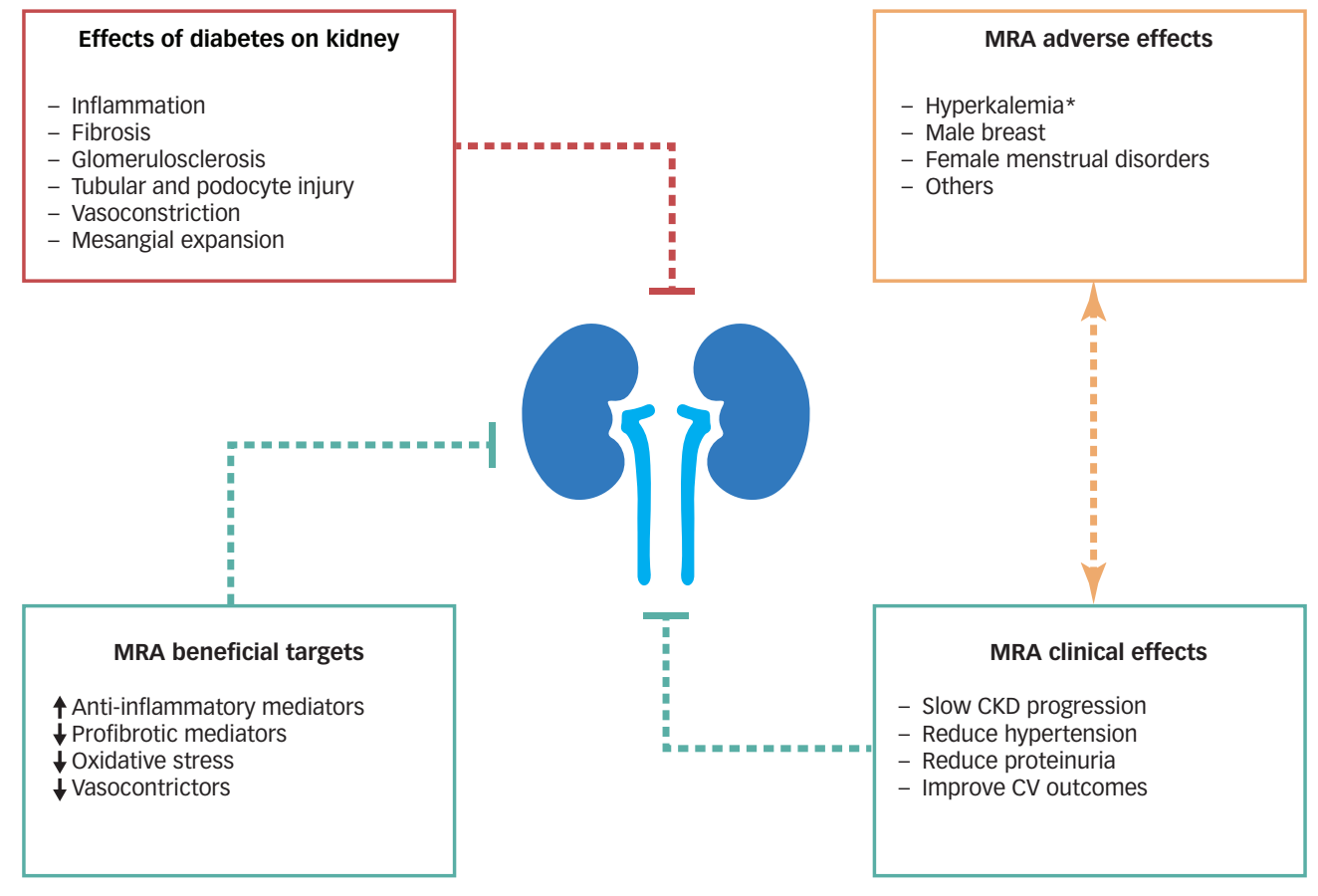

The adverse kidney effects of diabetes (in red) could be improved by the use of MRA through beneficial targets (in green), such as increased anti-inflammatory mediators and decreased pro-fibrotic mediators and oxidative stress, among others. These mechanisms may improve the renal function and slow CKD with additional CV benefits (in green). some warning of these agents (in orange) should be noted.

$C K D=$ chronic kidney disease; $C V=$ cardiovascular; $M R A=$ mineralocorticoid receptor antagonist .

*Associated more with steroidal MRAs.

\section{Diabetic kidney disease pandemic}

Worldwide, 463 million people, equivalent to 1 in 11 adults, are estimated to have T2DM. In Europe, almost 60 million people are affected by this disease, and in the USA 34.2 million, representing $10.5 \%$ of the country's population. ' Moreover, it is estimated that 232 million people have undiagnosed diabetes and 374 million people (1 in 13 adults) have impaired glucose tolerance. ${ }^{2}$ Thus, the clinical burden and socioeconomic impact of T2DM is huge, and the total annual medical and societal costs related to diabetic complications are estimated to increase to more than US $\$ 622$ billion by $2030 .^{2}$

A recently published large, retrospective, population-based cohort study analysed a total of 65,731 adults with T2DM and CKD, and revealed that CKD progression was observed in approximately $10-17 \%$ of patients over a median follow-up of 2 years. ${ }^{9}$ Similar prior studies showed that accelerated progression of kidney dysfunction leading to ESKD is more frequent in patients with diabetes compared with CKD from other aetiologies, such as proteinuria, heart failure, anaemia and elevated systolic blood pressure, all of which are predictors of fast progression. ${ }^{10}$ Diabetic-mediated kidney damage is currently detected and monitored by albuminuria, although more accurate biomarkers are needed. Apart from being a marker of kidney damage and progression, albuminuria is an independent predictor of all-cause mortality and cardiovascular events and mortality. ${ }^{11}$

Hyperglycaemia, low-grade inflammation, altered lipid metabolism, RAAS hyperactivation and increased sympathetic activity seem to be interrelated mechanisms contributing to both T2DM and microvascular complications. ${ }^{12-14}$ All these factors indirectly contribute to endothelial dysfunction, particularly that caused by oxidative stress linked to reduced nitric oxide bioactivity and leading to intraglomerular damage. ${ }^{9,13}$

Current treatments for DKD improve the pathological pathways involving RAAS activation, and metabolic and haemodynamic changes. In contrast, they do not produce a clear improvement on the pathways of inflammation, oxidative stress and fibrosis. New therapeutic options are needed to block these complementary pathogenic mechanisms in order to reduce residual renal risk and prevent the growth of the DKD pandemic.

\section{Mineralocorticoid receptor antagonist in chronic activation of the RAAS}

DKD progression is closely associated with chronic hyperactivation of the RAAS, and the role of MRAs in those patients affected has been rapidly acknowledged by the medical community owing to a strong physiological rationale, as mineralocorticoid receptors are widely expressed in different tissues such as kidneys and heart cells. The blockage of these receptors by MRA leads to haemodynamic and non-haemodynamic effects, resulting in a decrease in inflammation and fibrosis, and a slowdown in the progression of cardiorenal disease..$^{15}$

Regarding haemodynamic changes associated with finerenone, the actual evidence suggests that a decrease in intraglomerular pressure may limit podocyte injury and albuminuria, preventing albuminuria-induced tubular cell inflammatory and pro-fibrotic responses, thus preventing the loss of klotho, and even decreasing the metabolic load of proximal tubular cells. This pattern is consistent with the response to conventional RAAS blockers. ${ }^{16}$ 
Figure 2: Milestones in the development of mineralocorticoid receptor antagonists for the treatment of heart failure and diabetic kidney disease $7,8,17-20$

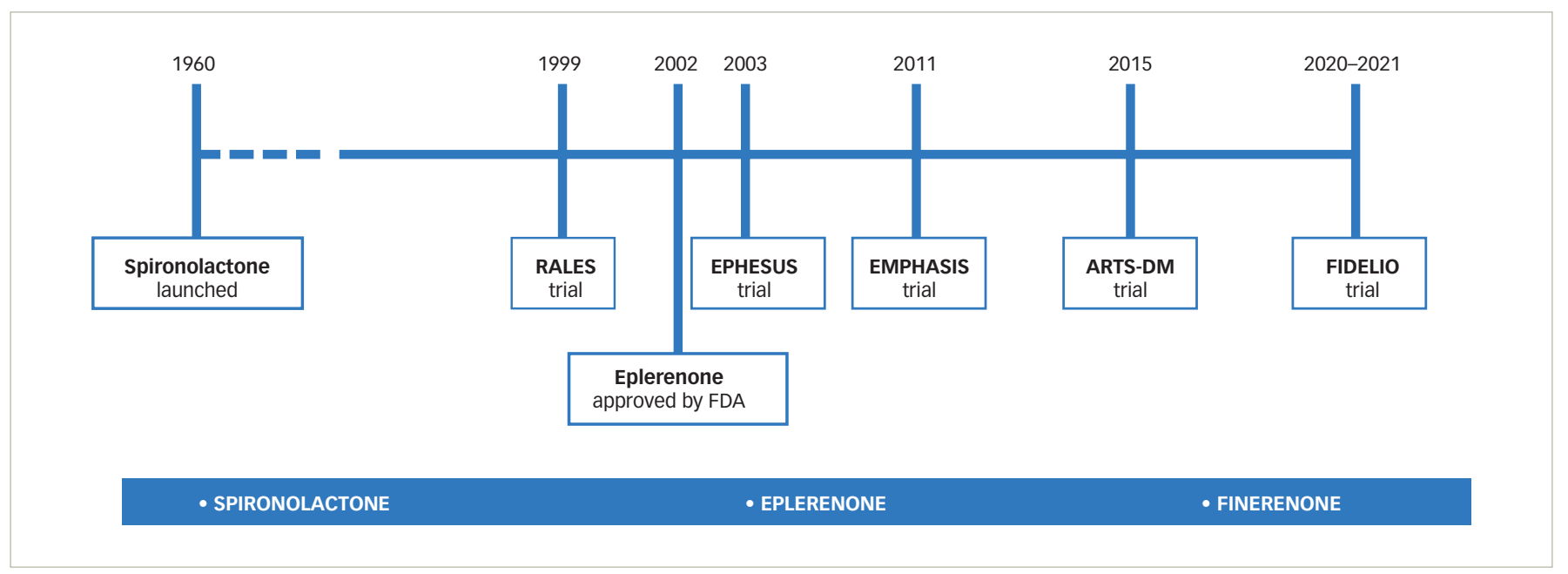

FDA = US Food and Drug Administration

Beyond the haemodynamic effects, finerenone has a direct action on tissue inflammation and fibrosis. These changes relate to inhibition of pro-inflammatory and pro-fibrotic effects recruited by the transcription factor induction via the mineralocorticoid receptor. Furthermore, mineralocorticoid receptor activation is a key mediator of renal damage induced by klotho deficiency and resulting from deficiency of klotho actions on adrenal cells, independently of RASS activation. ${ }^{16}$ Finally, these mineralocorticoid receptor activations are common with the activation of the angiotensin II/angiotensin receptor axis. ${ }^{15}$

\section{Mineralocorticoid receptor antagonists: Current perspective}

Steroidal MRAs have been used for decades for the treatment of hypertension, primary aldosteronism and heart failure (Figure 2). ${ }^{7,8,17-20}$ Oxidative stress, inflammation and kidney fibrosis are the common endpoints of CKD from various aetiologies, including DKD. Several experimental and clinical studies have shown that MRA improved kidney outcomes, such as decreased renal death or doubling of creatinine, reduced proteinuria, and neutral effects on estimated glomerular filtration rate (eGFR). ${ }^{21}$

Recent evidence supports the use of MRA drugs showing therapeutic benefits in renal outcomes. Since the release of data from the FIDELIO-DKD study ${ }^{7}$ and the preliminary report from the FIGARO-DKD trial ${ }^{8}$ researchers have focused attention on the selective nonsteroidal MRA, finerenone, and the capacity to improve the risk of CKD progression, with additional benefits of slowing down CVD progression in patients with CKD and/or T2DM. Indeed, it has been reported that finerenone reduced albuminuria after 4 months of treatment, with minimal effects on blood pressure and glycaemia. ${ }^{22,23}$

Steroid MRAs exhibit greater accumulation in kidneys than in the heart; in contrast, finerenone shows equal distribution in the heart and kidney. Of interest, the clearance of finerenone is predominantly mediated through non-kidney routes of elimination and without biologically active metabolites. ${ }^{24}$

A growing body of evidence from animal and preclinical studies suggests that finerenone is effective at slowing end-organ damage in cardiorenal disease. ${ }^{24,25}$ These favourable kidney effects are explained by downregulation of expression of proinflammatory and profibrotic markers, reduction in albuminuria and regression of tubulointerstitial fibrosis. Moreover, updated information about the renal activation of the aldosterone mineralocorticoid receptor system in the development of salt-sensitive hypertension and renal injury will provide new insights into the uses of MRAs for the treatment of cardiorenal diseases by different mechanisms. ${ }^{26}$ Similarly, in animal models of cardiac hypertrophy, finerenone was more effective at reducing cardiac fibrosis, plasma prohormone levels of brain natriuretic peptide (BNP) and albuminuria compared with eplerenone. ${ }^{21}$

Although the evidence from ongoing and new trials is awaited, existing studies may provide supporting data. The ROTATE-3 (Rotation for Optimal Targeting of Albuminuria and Treatment Evaluation) trial explores the different albuminuria-lowering drug classes to identify the individual drug response in diabetic and non-diabetic patients with CKD. ${ }^{27}$ This trial was designed to investigate the individual albuminuria response to an MRA (eplerenone) and an SGLT-2 inhibitor (dapagliflozin). Moreover, the Mineralocorticoid Receptor Antagonist Tolerability Study (ARTS) is a phase $\mathrm{llb}$ trial that was conducted to explore the safety and tolerability of finerenone relative to placebo or active treatment with spironolactone in patients with heart failure and in mild stages of CKD. ${ }^{17}$ Preliminary data report that events of hyperkalaemia or declined kidney function occurred less frequently in the finerenone arm than in patients randomly assigned to spironolactone. Finerenone and spironolactone appeared to be equally effective in improving albuminuria and the serum levels of $\mathrm{N}$-terminal pro-BNP (NTproBNP). ${ }^{22}$ These results will help to improve the current strategies for treatment and to slow DKD progression in those patients affected.

\section{Mineralocorticoid receptor antagonists: Warnings}

Steroid MRAs (spironolactone and eplerenone) can significantly improve the prognosis and quality of life in patients with heart failure, and reduce the rates of hospitalization and mortality. ${ }^{18}$ However, due to the low selectivity, these classic MRAs may lead to serum potassium elevation, development of gynaecomastia, female menstrual disorders and other undesirable effects, which limits their clinical use. ${ }^{21}$ However, recent evidence showed that finerenone had excellent selectivity to the mineralocorticoid receptor, and its half maximal inhibitory concentration $\left({ } C_{50}\right)$ was only $17.8 \mathrm{nmol} / \mathrm{L}$ (spironolactone $\mathrm{IC}_{50}=24.2 \mathrm{nmol} / \mathrm{L}$, eplerenone $\mathrm{IC}_{50}=990 \mathrm{nmol} / \mathrm{L}$ ). ${ }^{23}$ Thus, 
finerenone selectivity to mineralocorticoid receptor is significantly higher (>500-fold) than to glucocorticoid, androgen and progesterone receptors, respectively.28 Finally, close monitoring of potassium levels is warranted in those patients under renin-angiotensin system blockade and MRA treatments. Physicians should be aware of the risk of hyperkalaemia in all patients and especially in patients with eGFR $<30 \mathrm{~mL} / \mathrm{min} / 1.73 \mathrm{~m}^{2} .{ }^{29}$

With the focus still on hyperkalaemia risk, two recent alternative potassium-binding resins, patiromer sorbitex calcium and sodium zirconium cyclosilicate, have shown promising results in the management of patients predisposed to developing recurrent hyperkalaemia, and their use may allow for further optimization of guideline-directed medical therapy. ${ }^{30}$

\section{Combined drugs in the treatment of diabetic kidney disease}

Beyond the classic uses of RAAS blockade with angiotensin-converting enzyme inhibitors or angiotensin receptor blockers in the treatment of DKD progression, the combinations of SGLT-2 inhibitors, GLP-1 receptor agonists and nonsteroidal MRAs are expected to be increasingly used in the setting of DKD. In advance of these novel findings for finerenone in the cardiovascular field, current evidence supports the combined use of SGLT-2 inhibitors and GLP-1 receptor agonists to slow the progression of DKD, with renal and cardiovascular benefits. These drugs are proven to have an additive effect on lowering weight and blood pressure. It has also been suggested that the addition of SGLT-2 inhibitors and GLP-1 receptor agonists to insulin therapy could reduce the dose of insulin required, or even allow for its withdrawal, as well as achieving an ideal triad in the treatment of diabetes (good glycaemic control with no weight gain and reduced risk of hypoglycaemia), with the added cardiorenal benefits. ${ }^{31}$ As we commented earlier, the evidence for MRA is incomplete, pending the results from ongoing and new trials; however, future evidence could support the combined use of MRA with other drugs in the therapeutic arsenal for the management of DKD.

\section{Conclusion}

Novel MRAs have demonstrated efficacy in slowing the progression of CKD in patients with diabetes. Finerenone has a smaller effect on serum potassium levels than classic MRAs and a different side-effect profile to other agents, such as SGLT-2 inhibitors and GLP-1 receptor agonists, for the treatment of CKD in T2DM. Hence, combining RAAS blockade with the new hypoglycaemic agents such as SGLT-2 inhibitors, GLP-1 receptor agonist and finerenone will allow treatment of DKD by addressing several key factors in its progression, such as haemodynamic dysfunction, inflammation and fibrosis, with optimal and safe glycaemic control. Finally, although new evidence for finerenone in the cardiorenal field is pending, it is one of the most promising treatments for patients with DKD. $\square$
1. Rowley WR, Bezold C, Arikan Y, et al. Diabetes 2030: Insights from yesterday, today, and future trends. Popul Health Manas. 2017;20:6-12

2. Saeedi P, Petersohn I, Salpea P, et al. Global and regional diabetes prevalence estimates for 2019 and projections for 2030 and 2045: Results from the International Diabetes Federation Diabetes Atlas, 9th edition. Diabetes Res Clin Pract. 2019; 157:107843.

3. Buse JB, Wexler DJ, Tsapas A, et al. 2019 update to: Management of hyperglycaemia in type 2 diabetes, 2018. A consensus report by the American Diabetes Association (ADA) and the European Association for the Study of Diabetes (EASD). Diabetologia. 2020;63:221-8. Correction in: Diabetologia. 2020;63:1667.

4. Perkovic V, Jardine MJ, Neal B, et al. Canagliflozin and renal outcomes in type 2 diabetes and nephropathy. N Eng/ J Med. 2019;380:2295-306.

5. Agarwal R, Kolkhof P, Bakris G, et al. Steroidal and non-steroidal mineralocorticoid receptor antagonists in cardiorenal medicine Eur Heart J. 2021;42:152-61.

6. Liu LC, Schutte E, Gansevoort RT, et al. Finerenone: Third-generation mineralocorticoid receptor antagonist for the treatment of heart failure and diabetic kidney disease. Expert Opin Investig Drugs. 2015;24:1123-35.

7. Bakris $G L$, Agarwal R, Anker SD, et al. Effect of finerenone on chronic kidney disease outcomes in type 2 diabetes. N Engl J Med. 2020;383:2219-29.

8. Pitt B, Filippatos $G$, Agarwal R, et al. Cardiovascular events with finerenone in kidney disease and type 2 diabetes. N Eng/ J Med 2021. doi: 10.1056/NEJMoa2110956.

9. Carlström M. Nitric oxide signalling in kidney regulation and cardiometabolic health. Nat Rev Nephrol. 2021;17:575-90.

10. Go AS, Yang J, Tan TC, et al. Contemporary rates and predictors of fast progression of chronic kidney disease in adults with and without diabetes mellitus. BMC Nephrol. 2018;19:146.

11. Jankowski J, Floege J, Fliser D, et al. Cardiovascular disease in chronic kidney disease: Pathophysiological insights and therapeutic options. Circulation. 2021;143:1157-72.
12. Yang T, Richards EM, Pepine CJ, Raizada MK. The gut microbiota and the brain-gut-kidney axis in hypertension and chronic kidney disease. Nat Rev Nephrol. 2018;14:442-56.

13. Whaley-Connell A, Sowers JR. Basic science: Pathophysiology: The cardiorenal metabolic syndrome. J Am Soc Hypertens. 2014;8:604-6.

14. American Diabetes Association. 11. Microvascular complications and foot care: standards of medical care in diabetes - 2020. Diabetes Care. 2020;43(Suppl. 1):S135-51.

15. Lee JF, Berzan E, Sridhar VS, et al. Cardiorenal protection in diabetic kidney disease. Endocrinol Metab (Seoul). 2021;36:256-69.

16. Ortiz A, Ferro CJ, Balafa O, et al. Mineralocorticoid receptor antagonists for nephroprotection and cardioprotection in antagonists for nephroprotection and cardioprotection in Nephrol Dial Transplant. 2021;4;gfab167. doi: 10.1093/ndt/ Nephrol Dial

gfab167.

7. Pitt B, Anker SD, Böhm M, et al. Rationale and design of MinerAlocorticoid Receptor antagonist Tolerability Study-Hear Failure (ARTS-HF): A randomized study of finerenone vs. eplerenone in patients who have worsening chronic heart failure with diabetes and/or chronic kidney disease. Eur J Heart Fail. 2015;17:24-32.

18. Pitt B, Zannad F, Remme WJ, et al. The effect of spironolactone on morbidity and mortality in patients with severe heart failure N Engl J Med. 1999;341:709-17.

19. Pitt B, Remme W, Zannad F, et al. Eplerenone, a selective aldosterone blocker, in patients with left ventricular dysfunction after myocardial infarction. N Engl J Med 2003:348:1309-21.

20. Preiss D, van Veldhuisen DJ, Sattar $N$, et al. Eplerenone and new-onset diabetes in patients with mild heart failure: Results from the Eplerenone in Mild Patients Hospitalization and Survival Study in Heart Failure (EMPHASIS-HF). Eur $J$ Heart Fail. 2012;14:909-15.

21. Barrera-Chimal J, Girerd S, Jaisser F. Mineralocorticoid receptor antagonists and kidney diseases: pathophysiological basis. Kidney Int. 2019;96:302-19.

22. Agarwal $\mathrm{R}$, Anker $\mathrm{SD}$, Bakris $\mathrm{G}$, et al. Investigating new treatment opportunities for patients with chronic kidney disease in type 2 diabetes: The role of finerenone. Nephrol Dial Transplant. 2020;gfaa294. doi: 10.1093/ndt/gfaa294.

23. Ruilope LM, Tamargo J. Renin-angiotensin system blockade: Finerenone. Nephrol Ther. 2017;13(Suppl. 1):S47-53.

24. Orena S, Maurer TS, She L, et al. PF-03882845, a non-steroida mineralocorticoid receptor antagonist, prevents renal injury with reduced risk of hyperkalemia in an animal model of nephropathy. Front Pharmacol. 2013;4:115.

25. Huang $L L$, Nikolic-Paterson DJ, Han Y, et al. Myeloid mineralocorticoid receptor activation contributes to progressive kidney disease. J Am Soc Nephrol. 2014;25:2231-40

26. Ayuzawa N, Fujita T. The mineralocorticoid receptor in salt-sensitive hypertension and renal injury. J Am Soc Nephrol. 2021;32:279-89.

27. Provenzano M, Puchades M, Nicola L, et al. Study design of the rotation for optimal targeting of albuminuria and treatment evaluation (ROTATE-3): A rotation study of different albuminuria lowering drugs classes to study individual drug response in diabetic and non-diabetic CKD. Nephrol Dial Transplant. 2020;35(Suppl.):1003.

28. Pei H, Wang W, Zhao D, et al. The use of a novel non-steroida mineralocorticoid receptor antagonist finerenone for the treatment of chronic heart failure: A systematic review and meta-analysis. Medicine (Baltimore). 2018;97:e0254.

29. Navaneethan SD, Nigwekar SU, Sehgal AR, Strippoli GFM Aldosterone antagonists for preventing the progression of Aldosterone antagonists for preventing the progression of
chronic kidney disease: A systematic review and meta-analysis. chronic kidney disease: A systematic re

30. Gorriz JL, D'Marco L, Pastor-González A, et al. Long-term mortality and trajectory of potassium measurements following an episode of acute severe hyperkalaemia. Nephrol Dial Transplant. 2021 Jan 28:gfab003. doi: 10.1093/ndt/gfab003.

31. Morillas C, D'Marco L, Puchades MJ, et al. Insulin withdrawal in diabetic kidney disease: what are we waiting for? Int J Environ Res Public Health. 2021;18:5388. 